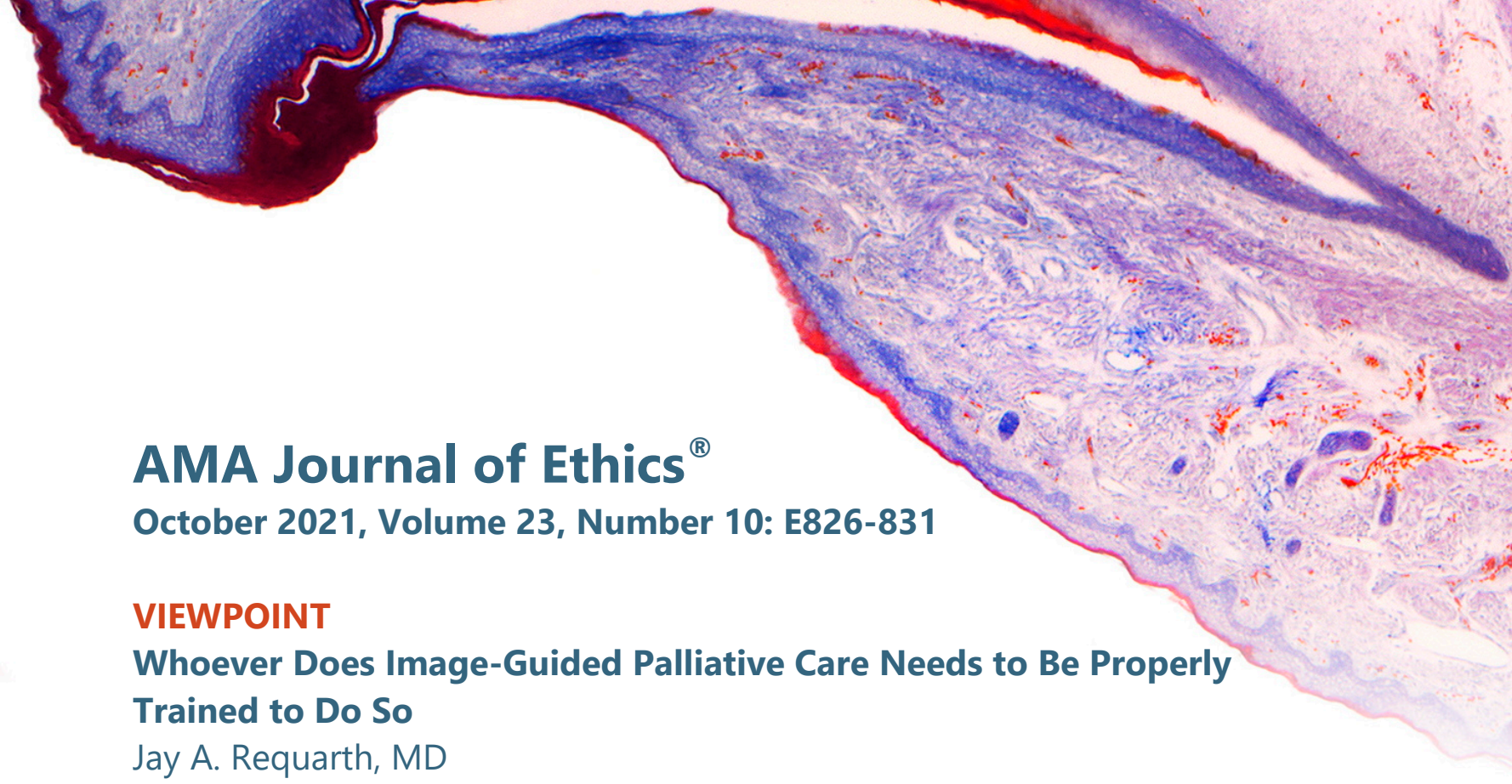

\begin{abstract}
Vascular and interventional radiologists (VIRs) often offer image-guided palliative care procedures, despite having little training in clinical medicine, let alone in palliative medicine. Informed consent tends to be inadequate, as does postprocedure patient care. This article proposes that VIRs who perform image-guided palliative procedures be sufficiently trained in palliative care or that surgeons or internists subspecialized in palliative care be sufficiently trained to provide image-guided techniques.
\end{abstract}

\title{
Image-Guided Palliation
}

Although hospice and palliative medicine is a recognized subspecialty, palliative and end-of-life care is provided by many different specialties. End-of-life and palliative care in the United States is fragmented, expensive, and often inconsistent with the patient's wishes. ${ }^{1}$ In my experience, nowhere are these limitations more apparent than in the image-guided palliative procedures provided by vascular and interventional radiologists (VIRs), many of whom have little training in clinical medicine, let alone in palliative medicine. ${ }^{2}$ Although historically, VIRs have championed new image-guided procedures such as angiography, endovascular stenting, embolotherapy, and endovascular thrombolysis, it wasn't until these procedures were adopted by specialties like cardiology and vascular surgery that they became mainstream. It is long past time either for VIRs to add palliative medicine to their armamentarium or to have physicians who provide palliative care learn VIR techniques. This article proposes that providing imageguided palliative care by specialists, similar to how care for atherosclerotic cardiovascular disease is provided by cardiologists, would substantially improve end-oflife and palliative care in the United States.

\section{What Went Wrong}

Over the years, much of my practice in surgery and interventional radiology gravitated toward palliative care procedures, and, having practiced hospice and palliative medicine (HPM), I used my HPM knowledge nearly every day. As examples of how diverse vascular and interventional radiological image-guided palliative procedures can be, I saw patients with liver failure and portal hypertension referred for transjugular intrahepatic portosystemic shunts, pelvic and bladder cancers referred for nephrostomy drain 
placement, biliary and pancreatic cancers referred for biliary drainage, and bleeding thoracic, abdominal, and pelvic cancers referred for embolotherapy. Furthermore, when I worked in hospice, I frequently saw patients who had undergone image-guided palliative procedures or should have undergone image-guided palliative procedures prior to their hospice referral.

During my decade as a surgeon working in vascular and interventional radiology, I was finally able to see inside the field's black box, and it was enlightening. Most, if not all, procedures performed in vascular and interventional radiology involve image-guided palliative care, ${ }^{2}$ which can benefit frail patients, patients with complex or hostile anatomy, or patients with advanced malignancies. However, in my experience, imageguided palliative procedures provided by VIRs are often painful and sometimes fail to achieve the desired clinical endpoint.

As a now-retired hospice and palliative care specialist, surgeon, and provider of VIR services, I also found the lack of pre- and postprocedural interactions distressing. I am not alone. About the then-new Practice Guideline for Interventional Clinical Practice, ${ }^{3}$ one reviewer stated:

It is bad medicine to perform an invasive therapeutic procedure on a patient without establishing the history, performing a physical examination, and developing or confirming a treatment plan with the patient ahead of time. It is unconscionable to perform that service and not follow the patient over time to see if it worked and if the patient is healthy and satisfied. ${ }^{4}$

VIRs' lack of patient interaction contributes to the public's impression that they are not physicians. In a 2018 survey, Heister and colleagues found that $83 \%$ of patients identified a urologist as a physician, but only $28 \%$ identified an interventional radiologist as a physician. ${ }^{5}$ Many VIRs have lamented this problem and urged their colleagues to become more visible by maintaining an inpatient practice, but this is not the norm.

\section{VIR Training}

As I have argued elsewhere, adding palliative care to VIR training would improve patient care and provide VIRs with necessary clinical skills to protect their turf.2,6,7 Despite recent changes in VIR training, 8 however, I have not seen progress toward adding palliative care education. Indeed, one qualitative study of 16 VIR fellows found that though they were more likely to be clinically oriented than their predecessors, $81 \%$ still did not consider clinical care to extend beyond the periprocedural period, and fellows who went into private practice found their clinical initiatives unsupported. 9

Since VIRs refuse or are unable to provide comprehensive palliative care, perhaps one solution is for physicians already familiar with diagnostic and palliative care to develop skill in image-guided procedures. Radiologists do not own the X-ray part of the electromagnetic spectrum. In fact, the transfer of image-guided procedures from VIRs to other clinicians is common and likely benefits patients. Coronary angiography, peripheral vascular angioplasty, dialysis access maintenance, and acute ischemic stroke treatment were all started by-or have been championed by-VIRs. But because VIRs failed to provide pre- and postprocedural care, skill in doing these procedures was developed by cardiologists, vascular surgeons, nephrologists, neurosurgeons, and neurologists. ${ }^{10,11,12,13,14,15}$ For example, between 1996 and 2007, the number of therapeutic endovascular procedures performed by vascular surgery resident physicians 
increased from an average of 7.2 to 103.6 .12 Most or all of these endovascular procedures were at one time performed by VIRs; now, they are almost exclusively performed by vascular surgeons and cardiologists uniquely qualified to provide the appropriate procedure and postprocedural care until a patient's death.

\section{Improving VIR Practice}

During informed consent, the burdens of an image-guided palliative procedure-not just the risks and benefits-need to be reviewed with a patient and his or her family by a skilled physician who will perform the procedure. Since quality of life should be a significant focus, postprocedural pain should be discussed and pain management options reviewed. Finally, discussion of a patient's do-not-resuscitate (DNR) order, if one exists, needs to be facilitated by the physician performing the procedure.

Informed consent. The physician has a duty to provide the patient with enough information for a reasonable patient to make an informed consent. ${ }^{16,17,18}$ Necessary components of informed consent include the name of the physician performing the procedure, diagnosis, intervention options, prospective risks and benefits, prognosis after the intervention or alternative interventions (including no intervention), chances of success, and recovery time. 16,17,18 Two informed consent cases adjudicated by state supreme courts, but not tested by the Supreme Court of the United States, suggest informed consent needs to be facilitated by the physician performing the procedure and given by a patient or appropriate surrogate before any intervention. ${ }^{19,20}$ To maximize palliative potential of an image-guided procedure, informed consent should be tailored to a specific patient at a specific point in time.

Informed consent should not be thought of as a process in which patient and physician set goals of care once and for all. Goals can change, and physicians need to ask about them every time they see a patient. For example, informed consent for percutaneous transhepatic drainage of unresectable obstructive jaundice should include informing a patient about how long the drain will be in place; pain associated with it; the possibility of hepatic artery injury; the need for frequent drain changes, if and when the drain can be removed; and, most importantly, the chance that the patient's serum bilirubin level will fall to a point at which palliative chemotherapy can start. Furthermore, the physician needs to understand the patient's wishes if complications develop. As objectionable as it might be to some physicians, there may be a day when the physician should allow the patient to die in the procedure room if things go terribly wrong.

Pain management. Informed consent should also include pain control. Even if a procedure is performed under general anesthesia or intravenous sedation, patients sometimes suffer from severe pain associated with follow-up procedures, such as drain changes. VIRs can ameliorate this pain, and they have an obligation to do so as best they can. Even if a patient is referred for a subcutaneous infusion port-catheter, VIRs can educate a patient about image-guided pain relief procedures, such as celiac plexus blocks for pancreatic cancer pain, during informed consent. For all patients, and especially for palliative care patients, if a procedure causes pain, sedation and intravenous opiates should be offered.

In my experience, pseudoaddiction-manifest when patients complain about pain before a procedure because they did not receive adequate pain control in prior procedural experiences-was common in drain exchange patients. As every palliative care specialist knows, pseudoaddiction is an iatrogenic problem caused by physicians undertreating 
severe pain. ${ }^{21}$ Often these patients are labeled as drug seekers or addicts, but they are not. Pseudoaddicts rarely ask for a prescription for pain medications for postprocedural pain control; they just want pain control during a procedure. Despite its importance in clinical care and despite the high likelihood of encountering patients with pseudoaddiction in vascular and interventional radiology, pseudoaddiction is not referenced in otherwise excellent textbooks or in Society of Interventional Radiology guidelines. ${ }^{3,22,23}$

DNR protocols. In my experience, VIRs often neither understand nor follow proper DNR protocols. ${ }^{16}$ Patients are not required to suspend their DNR order before a procedure. If significant complications occur during the procedure, a patient's death on the fluoroscopy table could be clinically and ethically appropriate. In my view, it is improper for VIRs (and anesthesiologists) to refuse to perform a procedure if patients choose not to suspend their DNR order during a procedure. In my experience, a patient's DNR order is routinely revoked by an anesthesiologist before a procedure because some anesthesiologists consider intravenous vasopressors to be a type of resuscitation. For this reason, I usually asked patients who did not want to suspend their DNR order to let me decide when resuscitation should stop. Obviously, this requires a significant amount of patient-physician trust, and I felt my palliative care knowledge helped me with these discussions. Finally, after surgery, when a patient is fully awake and can make decisions, the physician who performed the image-guided procedure should have another DNR discussion with the patient that includes information obtained during the procedure. This is also an excellent time to plan follow-up and pain management.

\section{What Palliative Patients Deserve}

Image-guided palliative procedures are performed by interventional radiologists, surgeons, pulmonologists, gastroenterologists, urologists, and other specialists, but follow-up with patients receiving these procedures is haphazard and often erroneously referred to interventional radiology. Many procedures non-VIRs now perform were pioneered by VIRs and appropriated by non-VIRs when the technique was perfected and billing problems resolved. In my experience, VIRs provide many procedures to palliative care patients but do not offer adequate informed consent, preprocedural care, and postprocedural care. Too often, a decision to offer image-guided palliative procedures and postprocedural care is left up to a referring physician.

Improving palliative and end-of-life care in the United States requires either that VIRs add palliative medicine to their training and take care of their patients before and after the procedure or that general surgeons and internists subcertified in HPM follow the cardiology model and learn to provide VIR skillfully. Patients deserve better than the status quo.

\section{References}

1. Institute of Medicine. Dying in America: Improving Quality and Honoring Individual Preferences Near the End of Life. National Academies Press; 2015.

2. McCullough HK, Bain RM, Clark HP, Requarth JA. The radiologist as a palliative care subspecialist: providing symptom relief when cure is not possible. AJR Am J Roentgenol. 2011;196(2):462-467.

3. American College of Radiology; American Society of Interventional and Therapeutic Neuroradiology; Society of Interventional Radiology. Practice guideline for interventional clinical practice. J Vasc Interv Radiol. 2005;16(2, pt 1):149-155. 
4. Murphy TP. American College of Radiology practice guideline for interventional clinical practice: a commitment to patient care. J Vasc Interv Radiol. 2005;16(2, pt 1):157-159.

5. Heister D, Jackson S, Doherty-Simor M, Newton I. An evaluation of trends in patient and public awareness of IR. J Vasc Interv Radiol. 2018;29(5):661-668.

6. Requarth JA. IR and palliative care: a good match. J Vasc Interv Radiol. 2015;26(11):1740-1741.

7. Requarth JA. Thinking very far outside the box: providing improved clinical care while ensuring admitting privileges by adding a clinical training experience in hospice and palliative medicine. J Vasc Interv Radiol. 2015;26(2)(suppl):S167S168.

8. IR residency and fellowship: becoming an interventional radiologist. Society of Interventional Radiology. Accessed November 30, 2020.

https://www.sirweb.org/learning-center/ir-residency/

9. Keller EJ, McGee KA, Resnick SA, et al. Who we are and what we can become: an analysis of professional identity formation in IR. J Vasc Interv Radiol. 2017;28(6):850-856.

10. Levin DC. The bygone era of interventional radiologists' involvement in coronary angiography. J Vasc Interv Radiol. 2019;30(11):1876-1877.

11. Hurks R, Ultee KHJ, Buck DB, et al. The impact of endovascular repair on specialties performing abdominal aortic aneurysm repair. J Vasc Surg. 2015;62(3):562-568.e3.

12. Mills JL Sr. Vascular surgery training in the United States: a half-century of evolution. J Vasc Surg. 2008;48(6)(suppl):90S-97S.

13. Murphy TP. Society of Interventional Radiology 2012 presidential address. $J$ Vasc Interv Radiol. 2012;23(8):987-988.

14. Moossavi S, Regan JD, Pierson ED, et al. Non-surgical salvage of thrombosed arterio-venous fistulae: a case series and review of the literature. Semin Dial. 2007;20(5):459-464.

15. Pierot L, Jayaraman MV, Szikora I, et al; Asian-Australian Federation of Interventional and Therapeutic Neuroradiology (AAFITN); Australian and New Zealand Society of Neuroradiology (ANZSNR); American Society of Neuroradiology (ASNR), et al. Standards of practice in acute ischemic stroke intervention: international recommendations. J Neurointerv Surg. 2018;10(11):1121-1126.

16. Requarth JA. Informed consent challenges in frail, delirious, demented, and donot-resuscitate adult patients. J Vasc Interv Radiol. 2015;26(11):1647-1651.

17. Ripley BA, Tiffany D, Lehmann LS, Silverman SG. Improving the informed consent conversation: a standardized checklist that is patient centered, quality driven, and legally sound. J Vasc Interv Radiol. 2015;26(11):1639-1646.

18. Berlin A, Kunac A, Mosenthal AC. Perioperative goal-setting consultations by surgical colleagues: a new model for supporting patients, families, and surgeons in shared decision making. Ann Palliat Med. 2017;6(2):178-182.

19. Annas GJ. Informed consent, cancer, and truth in prognosis. N Engl J Med. 1994;330(3):223-225.

20. Robeznieks A. Informed-consent ruling may have "far-reaching, negative impact." American Medical Association. August 8, 2017. Accessed November 28, 2020. https://www.ama-assn.org/practicemanagement/sustainability/informed-consent-ruling-may-have-far-reachingnegative-impact 
21. Weinstein S, Portenoy RK, Harrington SE, Bial A. UNIPAC 3: Assessing and Treating Pain. 4th ed. American Academy of Hospice and Palliative Medicine; 2012.

22. Kaufman EJ, Lee MJ. Vascular and Interventional Radiology: The Requisites. 2nd ed. Saunders Elsevier; 2014.

23. Valji K. Vascular and Interventional Radiology. 2nd ed. Saunders Elsevier; 2006.

Jay A. Requarth, MD is retired but maintains board certification by the American Board of Surgery and the American Board of Thoracic Surgery and is subcertified in hospice and palliative medicine by the American Board of Surgery. Following a fellowship year in vascular and interventional radiology (VIR), he worked over a decade in an academic VIR program.

\section{Citation}

AMA J Ethics. 2021;23(10):E826-831.

DOI

10.1001/amajethics.2021.826.

Conflict of Interest Disclosure

The author(s) had no conflicts of interest to disclose.

The viewpoints expressed in this article are those of the author(s) and do not necessarily reflect the views and policies of the AMA.

Copyright 2021 American Medical Association. All rights reserved. ISSN 2376-6980 